\section{Presencia y perspectivas de la enseñanza de la Historia reciente en la Historia enseñada en la educación secundaria en Chile. Un análisis desde el currículum}

Presence and perspectives of teaching recent history in the History curriculum in secondary education in Chile. A curriculum analysis

Gabriel Villalón-GálVeZ* Alicia Zamorano-VARGas ${ }^{* *}$

\section{Resumen}

En el presente artículo se presenta un análisis de la presencia y la perspectiva de enseñanza de la Historia Reciente en los currículos vigentes en la educación secundaria en Chile. Analizamos en particular cuál es la presencia de los temas de Historia Reciente en el currículo, qué tipo de temas son los tratados y cuál es la perspectiva que se vislumbra en el currículo. Para esto hemos analizado el Marco Curricular actualizado 2009 y las Bases Curriculares del año 2013. El análisis nos muestra que la Historia Reciente ha consolidado un espacio en el currículo de Historia en Chile y que la perspectiva que domina la enseñanza es la práctica.

Palabras clave: Historia Reciente, Enseñanza de la Historia, Currículum

\begin{abstract}
This paper examines the presence and teaching perspective of recent history in current curricula at secondary level in Chile. We analyse the presence of Recent History topics in the curriculum, what kind of topics are treated and what the possible perspective in the curriculum. For this, we analyse the 2009 Curricular Framework and the 2013 Curricular Bases. The analysis shows that recent history has consolidated a space in the curriculum of History in Chile and that the dominant perspective in teaching is the practical perspective.
\end{abstract}

Key words: Recent History, history teaching, curriculum

\footnotetext{
Departamento de Estudios Pedagógicos Facultad de Filosofía y Humanidades. Universidad de Chile, gabriel.villalon@uchile.cl

** Departamento de Estudios Pedagógicos. Facultad de Filosofía y Humanidades. Universidad de Chile, Alicia.zamorano@uchile.cl
} 


\section{La Historia, la escuela y la enseñanza de la Historia}

La relación entre Historia y Escuela concurren desde tiempos pretéritos, sin embargo será en la emergencia del surgimiento del Estado Nación en que este vínculo se consolidará. Esta relación se ha ido construyendo de forma vertical de manera que el saber histórico en la escuela ha estado sometido y ha sido utilizado por los intereses del Estado y en particular por los grupos de control y de influencia que ejercen el poder en él. Tal como plantea Cuesta (2000) la Historia en la Escuela ha abandonado el propio conocimiento histórico, para transformarse en una herramienta de la socialización ejercida por el Estado y el poder. En este sentido la Historia que se narra en las escuelas es resultado de intereses que se disputan la transmisión del pasado, para hacer entendible el presente. Como Carretero (2007) indica:

\begin{abstract}
La complejidad que caracteriza los procesos de construcción de las representaciones del pasado nos introduce en el problema de los usos de la historia públicos, políticos, populares y popularizados- y a entenderla no sólo como una disciplina, sino también como un tipo particular de gestión a favor de una memoria colectiva instituida e instituyente. En definitiva no se trata sólo de lo que se recuerda, sino también de lo que se olvida (Carretero 2007: 71).
\end{abstract}

La Historia en la escuela es el resultado de las representaciones que se hacen sobre el pasado en la sociedad, y que, según Carretero (2007) responden a tres tipos: la historia presente en la escuela a través de los currículos escolares, la historia cotidiana como parte de la memoria colectiva que articula los recuerdos, relatos de una sociedad o grupo humano constituyendo una serie de valores y creencias que les otorgan identidad; y la historia académica, que se refiere a la ciencia que desarrollan los historiadores o científicos sociales. Estos tres tipos de relatos históricos deben ser entendidos como narraciones que se encuentran interrelacionadas entre sí.

Entre estos tres tipos de narraciones destaca el de la Historia escolar que incluye una gran cantidad de valores que se enlazan en una trama de relatos cuya finalidad tradicionalmente ha sido la formación en los estudiantes de una visión positiva de la identidad de su nación. La escuela, tradicionalmente en el siglo XIX y en buena parte del siglo $X X$, ha transmitido un relato de la historia que tiene como finalidad principal la construcción de ciudadanos obedientes y comprometidos con la patria (Villalón y Pagés, 2015). Cuando la Historia en la escuela alcanza la categoría de disciplina y un lugar estable en los planes curriculares, adquiere características que casi no han variado desde el siglo XIX a la actualidad, a saber, la construcción de una narrativa elitista, arcaica, basada en la memorización y con fines nacionalistas. La Historia en la escuela tanto en sus contenidos, como en sus prácticas fue y ha sido una disciplina puesta al servicio de los discursos dominantes y una herramienta de socialización (Cuesta 2000; Villalón y Pagès 2013).

Estas características de la Historia escolar han comenzado a discutirse por su utilidad y su representatividad debido al avance de los procesos de escolarización universales. Los nuevos sistemas educativos permitieron colocar como principales destinatarios de la Historia escolar a los estudiantes pertenecientes a sectores tradicionalmente excluidos: pobres, pobladores, obreros, indígenas, negros, etc. De esta manera la Historia escolar caracterizada por ser monumental y anticuaria, comenzó a abrir espacio a una Historia Crítica que abandona los fines tradicionales. 
La transformación de la Historia Escolar se aceleró con la emergencia del surgimiento de las Didácticas Específicas, en este caso particular la Didáctica de la Historia (Benejam y Pagès 2004). El análisis crítico de la Historia escolar realizado desde la didáctica permitió distinguir la relevancia de las finalidades de la enseñanza. Investigaciones como las de Pagès (1994) o Thornton (1991) dieron cuenta que las prácticas pedagógicas de la Historia Escolar no eran únicas, sino que se concebían en una relación entre el currículum y sus objetivos, las instituciones y las finalidades de los docentes. En síntesis estas indagaciones permitieron relevar el rol que tiene el análisis de las finalidades del currículum y el rol de los docentes como un profesional que toma decisiones. En este contexto la Historia escolar y sus fines tradicionales asociados al poder y el Estado Nación era solamente una de las perspectivas de la Enseñanza de la Historia.

La Didáctica de la Historia ha identificado tres perspectivas de la Enseñanza de la Historia en relación con el currículum y sus finalidades: Tradicional, Práctica y Crítica (Goodman y Adler 1985; Grundy 1998; Pagès 1994; Benejam y Pagès 2004; Crowe, Hawley y Brooks 2012). La perspectiva tradicional sintetiza las características de la Historia Escolar por lo tanto sus finalidades son el mantenimiento del status quo, el desarrollo de los valores nacionales o construcciones de identidades excluyentes. En este contexto los contenidos históricos en la escuela son impuestos por las instituciones centrales del poder a través del Estado, que como finalidad principal tienen la socialización de los estudiantes a través de un relato romántico y lleno de progreso, por ejemplo: las historias nacionales oficiales. La transmisión de este relato histórico se encuentra en los distintos instrumentos curriculares y en el discurso de los docentes que son entendidos como técnicos repetidores o ejecutores de los lineamientos impuestos por el currículum preescrito. Finalmente, desde esta perspectiva se valora el aprendizaje cuando éste es capaz de memorizar y repetir el discurso oficial. Esta perspectiva de la enseñanza ha sido y es en buena parte la dominante en las aulas de Historia.

Las perspectivas prácticas y crítica de la enseñanza de la Historia surgen como una alternativa y respuesta a la perspectiva tradicional en tanto sus finalidades difieren y cuestionan la Historia escolar propia de la perspectiva tradicional. La perspectiva práctica de la enseñanza de la Historia coloca el acento en una construcción del aprendizaje de la Historia que busca que los estudiantes sean capaces de comprender el mundo que les rodea; el foco está en aprender Historia para desarrollarse personalmente y desde ahí actuar en la sociedad. Desde esta perspectiva la enseñanza abandona los objetivos socializadores del Estado Nación y de los grupos de poder, permitiendo dar énfasis al abordaje de contenidos que ayuden a los estudiantes a comprender su entorno. De ahí que las temáticas asociadas a la Historia de la comunidad y la memoria adquieren relevancia en esta perspectiva de la enseñanza (Pagès 1994).

La perspectiva crítica de la enseñanza de la Historia mantiene en sus objetivos que el aprendizaje histórico de los estudiantes les permita comprender su realidad inmediata y convertirse en ciudadanos preparados para transformar el mundo en el que viven. De esta manera la Historia enseñada considera el 
tratamiento de contenidos que sean relevantes socialmente. La Historia escolar desde esta perspectiva incorpora lo que Cuesta (2014) denomina una Historia Crítica y una Didáctica Crítica, donde esta última está constituida por tres componentes: a) Cultura: nada es lo que parece; b) Historia: genealogía del presente, y c) Política: poner a la cultura en su sitio. En esta línea la enseñanza de la Historia debe formar el pensamiento crítico y por lo que debe considerar el abordaje de temas controversiales en la escuela. La enseñanza de la Historia desde una perspectiva crítica considera el tratamiento de los conflictos sociales e históricos, las narrativas históricas sobres las y los subalternos, la memoria y la Historia Reciente permitiendo que adquieran un lugar central en la Historia escolar (Santisteban y Pagès 2011; Pagès y González 2009; Hess 2009; Hess y McAvoy 2015; Villalón y Pagès 2013; Villalón y Pagès 2015).

En síntesis, el desarrollo de la Didáctica de la Historia ha reconfigurado el rol de la Historia en la Escuela. Es así como en el siglo XXI los relatos históricos deben abandonar los objetivos de socialización del Estado Nación y los grupos de poder, para dar paso a una enseñanza que construya el conocimiento histórico, para que los ciudadanos construyan sus propias narrativas históricas a partir de la problematización del pasado y así ser ciudadanos constructores de una futura democracia. Por lo tanto, toma relevancia analizar cómo en la Historia escolar chilena se incorpora el tratamiento de cuestiones socialmente relevantes, como lo es la Historia Reciente, ya que la perspectiva de enseñanza presente en el currículum nos permiten tener una representación del tipo de ciudadanos que estamos formando en nuestras escuelas para el futuro.

\section{La Enseñanza de la Historia y la Historia reciente}

La emergencia del cambio social y las demandas de los nuevos actores sociales ha tenido como resultado la transformación, al menos en lo declarativo, de la Historia en la escuela. Hoy en día las finalidades de la Historia escolar suelen estar vinculadas a conseguir la formación de ciudadanos capaces de pensar críticamente y con el objetivo de que sean agentes activos en las decisiones políticas de la vida en democracia. Resultado de lo anterior tanto la Historia en la escuela, como la Enseñanza de la Historia ha transitado a la incorporación de nuevas temáticas en el aula escolar, de ahí distintas iniciativas y proyectos que han considerado incluir como contenidos problemas sociales relevantes, temas controversiales, la Historia de grupos subalternos, los conflictos, los Derechos Humanos y la Historia Reciente.

Las consecuencias de la Gran Guerra en Europa, las luchas de la descolonización en África y Asia, y los traumas de las dictaduras cívicomilitares en América Latina han colocado como un tema recurrente en las nuevas propuestas curriculares a la Historia reciente y la memoria. Sin embargo, el abordaje de la Historia reciente significa asumir una enseñanza de la Historia que construye aprendizajes a partir del tratamiento y análisis de los conflictos. El pasado reciente significa entender el aula de Historia como un espacio que aprende y se desarrolla en el análisis de los conflictos. Por esto, un desafío de enseñar la Historia reciente en la escuelas es el hecho que el pasado inmediato es un espacio en disputa en el que las fronteras del análisis no están aún definidas, es lo que los franceses han denominado cuestiones socialmente vivas (Tutiaux-Guillon 2011; Veneros 2014). 
Enseñar Historia reciente en la escuela significa confrontar una serie de problemáticas, ya que cuando enseñamos el pasado reciente nos vinculamos por lo general a cuestiones conflictivas, a experiencias que suelen tener una carga importante de violencia y dolor. Por lo tanto, cuando la Historia en la escuela quiere abordar los sucesos recientes debe tener en cuenta que trabaja con pasados vividos que se resisten a ser "pasados" (Kriger 2011). Es por esto que la Historia reciente ha tenido escasa presencia en la Historia enseñada en las escuelas. Por ejemplo en una revisión de los contenidos históricos de los textos escolares argentinos las conclusiones indicaban que la Historia en la escuela se concentraba fundamentalmente en la Colonia, la Independencia y el análisis del siglo XIX y la primera parte del siglo XX, incluso por un largo tiempo los textos se caracterizaban por no abordar más allá de la primeras décadas del siglo XX (Finocchio 2009). Ante esta situación Carretero y Borreli (2010) indican que la escuela tiene un rol importante en la transmisión del pasado reciente y en la comprensión crítica y activa de la Historia. Sin embargo, debe tener conciencia de las dificultades que enfrenta al abordar contenidos históricos recientes que son controvertidos para la comunidad.

Los desafíos y controversias con los que se encuentra la enseñanza de la Historia reciente en la escuela se explica por el desarrollo reciente que tiene en la historiografía este campo de estudio. Para la investigación histórica el estudio del pasado reciente continua siendo un ejercicio por consolidar (Carretero y Borreli 2010). Sin embargo, más allá de los desafíos y problemáticas que significa la incorporación la Historia reciente en el aula, hoy en día es cada vez más cotidiano encontrar propuestas curriculares y extracurriculares que incorporan estos temas en la Historia escolar. En principio organizaciones vinculadas a movimientos de memoria y derechos humanos y luego grupos de docentes han colocado las temáticas del pasado reciente en las aulas. Junto a esto desde la institucionalidad las reformas curriculares han incorporado contenidos relacionados con la Historia reciente.

En correspondencia con lo anterior hoy día es relevante examinar cuáles son perspectiva de la enseñanza de la Historia reciente en el currículum de la Historia enseñada en Chile. Esto porque la enseñanza de la Historia reciente es un contenido controversial que puede ayudar a los docentes a generar en los estudiantes los aprendizajes para el desarrollo del pensamiento histórico para la construcción de ciudadanías críticas (Pagès 2009; Levesque 2009).

\section{Presencia y perspectivas de la Enseñanza de la Historia Reciente en el currículum de Historia en Chile}

\subsection{Presencia de la Historia Reciente en el currículum de Historia en Chile}

La inclusión de los temas de la Historia Reciente en el currículum chileno se ha iniciado a partir de las reformas curriculares desarrolladas en el marco de los nuevos gobiernos democráticos de la llamada Transición chilena. La Historia Reciente de Chile en particular los procesos históricos sucedidos posteriormente al Golpe de Estado de 1973 aparecieron en la escuela con la Reforma educacional de mediados de los años 90 (Valencia 2013). Esta incorporación se desarrolló en el marco de tensiones en la academia, la opinión pública, y marcada por la política de los acuerdos característica de la 
democracia chilena de los años 90'. A partir de este momento y de manera permanente los temas de la Historia Reciente han tenido una permanencia constante en el currículum chileno.

En el Marco Curricular ajustado y en las nuevas Bases Curriculares se da cuenta de intenciones y objetivos que hacen coherente la incorporación de las temáticas de la Historia Reciente en el currículum escolar. Para el caso del Ajuste Curricular del año 2009 la incorporación de la Enseñanza de la Historia Reciente va en directa relación con los principios que orientan a la propuesta en torno al valor de la formación de ciudadanos para la vida en democracia. Esto se manifiesta en los Objetivos Fundamentales Transversales, por ejemplo en al ámbito de la Formación Ética se indica que se busca que:

\section{Alumnos y alumnas desarrollen y afiancen la voluntad para autorregular su conducta y autonomía en función de una conciencia éticamente formada en el sentido de su trascendencia, su vocación por la verdad, la justicia, la belleza, el bien común, el espíritu de servicio y el respeto por el otro (MINEDUC 2009).}

En una perspectiva similar en el sector de Historia, Geografía y Ciencias Sociales se indica de la relevancia del conocimiento histórico para comprender la realidad actual, en particular se espera que:

Alumnos y alumnas desarrollen sentido cívico, conozcan y comprendan los derechos y deberes que implica la vida en democracia, incluyendo la participación responsable en las actividades comunitarias y ciudadanas, que reconozcan la legitimidad de distintos puntos de vista, los que reflejan la diversidad de visiones que conviven en el país, que actúen responsablemente consigo mismos, con su entorno y en la sociedad, y que valoren los principios fundamentales de libertad, igualdad, justicia, pluralismo, responsabilidad social y respeto a los derechos humanos (MINEDUC 2009).

En el caso de las Bases Curriculares (Decreto Supremo de Educación n 614/2013) para los niveles de $7^{\circ}$ básico a $2^{\circ}$ medio continúa en una tendencia similar al currículum anterior, en cuanto a que tiene como finalidad la formación de ciudadanos activos y constructores de la democracia. En particular en las orientaciones sobre el aprendizaje se indica en el criterio número cinco que los alumnos necesitan comprender y apreciar los fundamentos de una sociedad democrática y libre y del papel de sus instituciones, para que puedan así participar responsable y activamente en la vida democrática. Para ello, es necesario que comprendan los hitos, procesos e ideas fundamentales de la historia de Chile y de la humanidad que han tenido relevancia para la conformación actual de la sociedad chilena (MINEDUC 2016).

En la asignatura de Historia, Geografía y Ciencias Sociales se propone la formación de ciudadanos activos y con una conciencia ética basada en los Derechos Humanos, de ahí la importancia de la formación de un pensamiento histórico que les permita mejor comprender su presente, para establecer conexiones con el pasado y trazar planes de futuro (MINEDUC 2016). La revisión del Marco Curricular y las Bases Curriculares nos muestra que en sus propósitos, fundamentos y principios consideran elementos que permiten y hacen coherente la incorporación de los temas vinculados a la Historia Reciente chilena y del mundo.

En cuanto al Marco Curricular ajustado 2009 los temas de la Historia Reciente encuentran presencia solamente en los cursos de $1^{\circ}$ y $3^{\circ}$ medio, esto se distribuye en cuanto a los Objetivos Fundamentales un $18,75 \%$ se relacionan con la Historia Reciente. En el caso de los ejes de Contenidos Mínimos e 11,90\% de los temas revisados entre $7^{\circ}$ año básico y $4^{\circ}$ 
año medio se relaciona con la Historia Reciente de Chile o el mundo. Para el caso de las Bases Curriculares la revisión nos muestra que los objetivos de aprendizajes y organizadores temáticos en relación con la Historia Reciente se encuentran solamente en el curso de $2^{\circ}$ año medio. Lo anterior significa que un $14,7 \%$ de los Objetivos de Aprendizajes y un 13\% de los Organizadores Temáticos de la Educación Media común ( $7^{\circ}$ a $2^{\circ}$ medio) se vincula con los temas de la Historia Reciente. La revisión de los currículos vigentes en la educación chilena comprueba lo indicado sobre que la Historia Reciente ha consolidado su presencia en los objetivos y contenidos de la enseñanza de la Historia en Chile.

\subsection{Qué se enseña sobre Historia Reciente en el currículum de Historia en Chile}

Una segunda cuestión a revisar sobre los currículos y la Historia Reciente es conocer qué es lo que se propone enseñar en cuanto a contenidos. En el caso del Marco Curricular Ajustado 2009 los contenidos que hemos identificados relacionados con la Historia Reciente se ubican en dos grandes grupos. El primer grupo vinculado a la Historia Mundial relacionada con la Historia de Europa y Estados Unidos durante la Guerra Fría esto es tratado durante el primer año medio. El segundo grupo se refiere a la Historia de Chile y se concentra en el análisis de la segunda mitad del siglo XX chileno resaltando los procesos de cambios acaecidos en los 50`y 60’, la revisión del quiebre de la democracia y el final abrupto del proyecto de la Unidad Popular, y para finalizar la Dictadura Militar (llamado Régimen Militar por el currículo) y el retorno a la Democracia, este segundo grupo de contenidos se concentra en el tercer año medio (MINEDUC 2009). Estos dos grandes grupos se pueden desglosar en 21 temas, que pueden revisarse en la tabla 1 de los anexos. Entre estos 21 temas destacamos los siguientes:

\footnotetext{
-Recuperación de testimonios y de expresiones de la literatura y las artes para describir las transformaciones sociales y culturales en Chile durante las décadas de 1960 y 1970.

-Confrontación de visiones políticas sobre la crisis que desemboca en el quiebre democrático de 1973.

-Caracterización de los principales rasgos del golpe de Estado y de la dictadura militar en Chile: la violencia política; la supresión del Estado de Derecho; la violación sistemática de los Derechos Humanos; la transformación neoliberal de Chile (la transformación del rol del Estado y la nueva política económica); la creación de una nueva institucionalidad política bajo la Constitución de 1980; las relaciones con los países vecinos y con la comunidad internacional.

-Valoración de la lucha por la defensa de los Derechos Humanos y la recuperación de la democracia (MINEDUC 2009).
}

En el análisis de los 21 sub-temas identificados el abordaje de la enseñanza de la Historia Reciente se encuentra dominada por contenidos de tipo tradicional, por esto nos referimos a aquellos que se refieren a la política tradicional, el desarrollo del Estado, la economía y el análisis de la sociedad en términos generales. Esto se manifiesta en que entre los 21 sub-temas encontramos que los contenidos que alcanzan mayor presencia son: contenidos sobre política tradicional (62\%); contenidos sobre el Estado (48\%); contenidos sobre economía (29\%); contenidos sociales $(33 \%) y$ cambios sociales $(29 \%)$. Es interesante mencionar que entre los contenidos que tienen mayor presencia aparecen aquellos relacionados con contenidos sobre ciudadanía (33\%). En el caso de los contenidos vinculados a la política tradicional estos aparecen en relación con los problemáticas de los bloques en conflicto durante la Guerra Fría o el análisis del rol de los partidos políticos antes, durante 
y posterior a la Dictadura Militar Chilena. En cuanto a los contenidos sobre Economía estos dice relación con los ciclos económicos y las respectivas crisis, así como también los modelos económicos preponderantes, por ejemplo el neoliberalismo. Los contenidos sobre el Estado se refieren fundamentalmente al desarrollo institucional de este y sus implicancias en el desarrollo económico y social, como por ejemplo el conocimiento de las características del Estado Bienestar. En cuanto a los contenidos sobre cuestiones sociales estos se refieren a las caracterizaciones que se realizan sobre la sociedad en su conjunto en cuanto a datos demográficos, aspectos culturales, surgimiento de nuevos grupos sociales, condiciones de vida, etc. Finalmente los contenidos que se vinculan a ciudadanía se concentran en cuestiones sobre el desarrollo de la participación política y a las contiendas por la recuperación de la democracia en Chile y Latinoamérica.

Con una menor presencia en la propuesta del Marco Curricular 2009 se encuentra el tratamiento de contenidos sobre cuestiones como: Los Derechos Humanos (19\%), Dictadura (14\%), Revoluciones (10\%), Cambios Tecnológicos (10\%), Subalternos (5\%), movimientos sociales (5\%), procesos de descolonización (5\%). Adquiere notoriedad en este segundo grupo de contenidos identificados la inclusión del tema de Derechos Humanos que aparecen vinculados primero en un análisis del terrorismo de Estado en América Latina y otras regiones del mundo. Los contenidos vinculados a Derechos Humanos aparecen luego vinculados al accionar del Estado durante la Dictadura en Chile y a los movimientos en defensa de los Derechos Humanos.

En el caso de las Bases Curriculares los contenidos que hemos identificado relacionados con la Historia Reciente se desarrollan durante el segundo año medio. En una primera parte se revisan aspectos de la Historia Mundial con el objetivo de que se busca que los:

Estudiantes analicen la Guerra Fría y los diversos escenarios en que se manifiesta y, asimismo, los procesos que marcan el cambio de un mundo bipolar a uno globalizado hacia fines del siglo. En este marco, interesa analizar cómo la Guerra Fría impactó en América Latina y generó una tensión permanente entre revolución y reforma que desembocó en golpes de Estado y dictaduras militares en distintos países del continente (MINEDUC 2016: 181).

En una segunda parte se analiza:

Segunda mitad del siglo XX chileno a partir de las reformas estructurales, las nuevas demandas sociales $y$ la polarización política. Junto a esto, se busca reconocer cómo el ambiente de crisis de fines de los '60 y principios de los '70 desembocó en el quiebre de la democracia. Respecto de la dictadura militar interesa que los estudiantes reconozcan que se suprimió el Estado de derecho y que se violaron sistemáticamente los Derechos Humanos, y que analicen las transformaciones que provocaron en la sociedad chilena la implantación de un nuevo modelo económico y de una nueva institucionalidad política. Asimismo, se estudiará el proceso de recuperación de la democracia, y las transformaciones sociales y culturales de la sociedad chilena a partir de 1990 (MINEDUC 2016: 182)

Estos contenidos se desarrollan en tres organizadores temáticos: "De un mundo bipolar a un mundo globalizado: la Guerra Fría"; "Chile en el contexto de la Guerra Fría: transformaciones estructurales, polarización política y social y quiebre de la democracia"; y "Dictadura militar, transición política y consenso en torno a la democracia en el Chile actual", en estas tres unidades se propone desarrollar 14 objetivos de aprendizajes con sus respectivos contenidos. A continuación mostramos algunos de los objetivos de aprendizaje vinculados a la Historia Reciente chilena y mundial:

-Reconocer las transformaciones que experimentó la sociedad occidental en diversos ámbitos durante la 
Guerra Fría, como por ejemplo el crecimiento económico y el auge del Estado de Bienestar, la expansión del consumo y de los medios de comunicación de masas, la ampliación de los derechos civiles de grupos marginados, el desarrollo tecnológico, entre otros.

-Caracterizar el contexto de movilización social en América Latina como un escenario de tensión permanente entre revolución y reforma, considerando la Revolución Cubana, la influencia de Estados Unidos, los golpes de Estado, las dictaduras militares (por ejemplo, Argentina, Chile, Brasil, Uruguay y Paraguay, entre otros), y la violación de los Derechos Humanos.

-Analizar los principales procesos que marcaron el fin de la Guerra Fría, considerando la crisis de la URSS y el derrumbe del comunismo, las transformaciones geopolíticas, el auge del neoliberalismo, y la aceleración de la globalización (por ejemplo, la internacionalización de la economía, la revolución tecnológica, la sociedad de la información). Chile en el contexto de la Guerra Fría: transformaciones estructurales, polarización política y social y quiebre de la democracia.

-Analizar el Chile de la década de 1960 como un escenario caracterizado por la organización y la movilización de nuevos actores sociales (por ejemplo, jóvenes, campesinado, pobladores), y evaluar cómo el sistema político respondió a estas demandas, considerando las reformas estructurales y los proyectos excluyentes de la Democracia Cristiana ("revolución en libertad") y de la Unidad Popular ("vía chilena al socialismo").

-Analizar el ambiente de crisis a inicios de la década del '70, considerando aspectos como la polarización social y política, la retórica de la violencia, la desvalorización de la institucionalidad democrática, los conflictos en torno a la reforma agraria, las estatizaciones y expropiaciones, la crisis económica y la hiperinflación, la movilización social, la intervención extranjera y el rol de las Fuerzas Armadas. -Analizar y comparar críticamente distintas interpretaciones historiográficas sobre el golpe de Estado de 1973 y el quiebre de la democracia. Dictadura militar, transición política y consenso en torno a la democracia en el Chile actual.

-Explicar que durante la dictadura militar se suprimió el Estado de Derecho y se violaron sistemáticamente los Derechos Humanos, reconociendo que hubo instituciones civiles y religiosas que procuraron la defensa de las víctimas.

-Explicar los factores que incidieron en el proceso de recuperación de la democracia durante la década de 1980, considerando la crisis económica, el surgimiento de protestas, la rearticulación del mundo político y el rol mediador de la Iglesia Católica, de organismos de defensa de Derechos Humanos y de la comunidad internacional. -Analizar la transición a la democracia como un proceso marcado por el plebiscito de 1988, la búsqueda de acuerdos entre el gobierno y la oposición, las reformas constitucionales, las tensiones cívico militares, el consenso generado en torno a la democracia representativa como sistema político y la reivindicación de los Derechos Humanos mediante diversas políticas de reparación (MINEDUC 2016: 210-212).

El análisis de los contenidos desarrollados en los objetivos de aprendizaje indicados anteriormente muestra que para el caso de las Bases Curriculares los contenidos que tienen mayor presencia son los referidos a la Política tradicional (50\%), Economía (50\%), Ciudadanía (50\%) y Estado (36\%). Como se puede observar la enseñanza de la Historia reciente en las Bases Curriculares 2013 mantiene la tendencia de concentrarse en el tratamiento de temas tradicionales como los políticos y económicos. Los contenidos referidos a las cuestiones políticas se concentran en una primera parte en los procesos asociados a la Guerra Fría. Una segunda parte se concentran fundamentalmente en el desarrollo político chileno con énfasis en el rol de los partidos políticos y otras organizaciones antes, durante y posterior a la Dictadura. Una cuestión que destaca en las Bases Curriculares es la presencia que adquieren los contenidos vinculados a la ciudadanía. Los contenidos asociados a estas temáticas hacen referencia principalmente a ganancias y pérdidas en relación con la democracia, luchas por la ampliación de derechos y análisis de la institucionalidad impuesta en dictadura y luego reformada en democracia en Chile.

En el caso de las Bases Curriculares encontramos un segundo grupo de temas con una presencia significativa en relación con las temáticas de la Historia Reciente, aquí se identifican los siguientes temas: subalternidades (29\%), movimientos sociales (29\%), cambios sociales (29\%), dictaduras 
(29\%), derechos humanos $(21 \%)$ y cuestiones sociales (21\%). En este segundo grupo destacan la incorporación de los temas sobre subalternidades y movimientos sociales, los contenidos que se desarrollan proponen revisar el rol de organizaciones sociales en las distintas problemáticas política y sociales de la segunda mitad del siglo XX. Finalmente encontramos un tercer grupo por contenidos con una presencia menor, estos temas son cambios tecnológicos $(14,30 \%)$, procesos de descolonización (7\%) y procesos revolucionarios (7\%).

El análisis de los contenidos a enseñar en el Marco Curricular ajustado 2009 y la Bases Curriculares 2013 continúa manteniendo un predominio de presencia de temas de tipo tradicional como los políticos y económicos. Sin embargo, en los dos currículos revisados se nota la inclusión de nuevas temáticas que comienzan a tener mayor presencia entre los contenidos, un primer caso son los temas asociados al desarrollo de la ciudadanía. En las Bases Curriculares se observa una consolidación del tratamiento de nuevos temas como los asociados a movimientos sociales, subalternidades, dictaduras y derechos humanos. En conclusión podemos establecer que la enseñanza de la Historia Reciente, según lo analizado en los currículos, tiene como característica la convivencia entre contenidos de tipo tradicional y la incorporación de nuevas temáticas socialmente relevantes.

\subsection{Perspectiva de la Enseñanza de la Historia Reciente en el currículo chileno}

La Historia Reciente ha consolidado un espacio en el currículo chileno de Educación Media. Como hemos visto, tanto el Marco Curricular Ajusta 2009 y las Bases Curriculares 2013, incorporan objetivos y contenidos en el área, sin embargo, queda por analizar si la presencia estable y la incorporación de objetivos y contenidos sobre Historia Reciente significan además una perspectiva de la enseñanza de la Historia que abandone las prácticas tradicionales (Pagès 1994).

Como hemos indicado anteriormente la Historia escolar ha tenido como característica el desarrollo de una perspectiva técnica en concordancia con los fines de los Estados Nacionales y los grupos de poder por utilizar la escuela como un espacio de socialización del sistema. Este discurso se caracterizó por la imposición de una educación cívica con finalidades de formar ciudadanos pasivos, la instalación de los valores patrios en los estudiantes y la enseñanza de una Historia que diera cuenta de las hazañas de la nación y del progreso continuo de esta (Villalón y Pagès 2015). Esta perspectiva dominante de la Enseñanza de la Historia desde el siglo XIX ha sido contrarrestada por el desarrollo de otros tipos de prácticas como las perspectivas prácticas y críticas. Estas perspectivas colocan un fuerte énfasis en el desarrollo de una enseñanza para la vida en democracia (Pagès y Benejam 2004). Ambas perspectivas pese a tener bastantes similitudes difieren en su finalidad. La perspectiva práctica entiende que la enseñanza de la Historia debe contribuir al desarrollo personal de los sujetos y desde ahí construye los contenidos y objetivos del currículo. Por su parte, la perspectiva crítica entiende que si se enseña Historia para la vida en democracia, se debe tener como finalidad que los sujetos adquieran los saberes que les permitan ser agentes transformadores (Pagès 1994; Cuesta 2014).

Los propósitos, contenidos mínimos y objetivos fundamentales del Marco Curricular 2009 
dan cuenta de una perspectiva práctica de la enseñanza en la que el desarrollo personal del estudiante es la finalidad de la práctica educativa. Esto se hace evidente en la declaración de que "alumnos y alumnas desarrollen y afiancen la voluntad para autorregular su conducta y autonomía en función de una conciencia éticamente formada" (MINEDUC 2009). En una línea similar continúan los propósitos del sector de Historia, Geografía y Ciencias Sociales cuando declaran que los estudiantes conozcan derechos y deberes de una vida en democracia (MINEDUC 2009). La claridad de una perspectiva práctica de la enseñanza de la Historia se hace menos clara cuando el análisis recae sobre los objetivos y contenidos específicos que hemos identificado con la Historia Reciente. En primer lugar se puede observar que los contenidos y objetivos colocan como valor permanente el análisis de procesos históricos que tienen relación con la construcción de derechos y la democracia, como se puede ver en el caso de los siguientes contenidos mínimos de $3^{\circ}$ medio:

-Descripción de la masificación de la participación política popular y caracterización de la creciente demanda de cambio social.

-Valoración de la lucha por la defensa de los Derechos Humanos y la recuperación de la democracia.

-Contextualización del proceso político chileno en el marco de las dictaduras y la recuperación de la democracia en el Cono Sur (MINEDUC 2009: 236-237).

La concordancia de los contenidos sobre Historia Reciente con una perspectiva práctica de la enseñanza se hace menos clara, primero cuando la revisión de cuáles son los temas que se propone enseñar nos muestra que la mayor parte son sobre política tradicional (62\%) y contenidos sobre el Estado (48\%). Una segunda cuestión que muestra un alejamiento de la perspectiva práctica de la enseñanza de la Historia en los contenidos es el que no se logra distinguir en éstos la intención que los estudiantes aprendan desde su realidad inmediata. La propuesta de contenido mínimos propone un análisis de la Historia Reciente o desde una perspectiva país o nacional y no existen entre los contenidos una mención al estudio de la realidad histórica inmediata de los estudiantes.

Las Bases Curriculares también muestran elementos propios de una perspectiva práctica de la enseñanza de la Historia. En los propósitos se declaran por el desarrollo de un aprendizaje para la vida en democracia, se indica que los saberes adquirir a través del proceso educativo deberán a ayudar a los estudiantes a realizar su "potencial y vivir su vida en forma plena, participando activamente en una sociedad libre, democrática y pluralista, y contribuyendo responsablemente al desarrollo del país" (MINEDUC 2016: 13). Esto es reafirmado en la asignatura de Historia, Geografía y Ciencias Sociales. La perspectiva práctica en relación a la enseñanza de la Historia Reciente en el caso de las Bases Curriculares se hace notar en los contenidos que se desarrollan. Esto se observa en la presencia mayor de contenidos que en el currículo anterior de contenidos referidos a cuestiones ciudadanas y actores históricos no tradicionalesymovimientossociales, porejemplo los contenidos referidos a subalternidades (29\%), movimientos sociales (29\%), cambios sociales $(29 \%)$, dictaduras $(29 \%)$, derechos humanos $(21 \%)$ y cuestiones sociales $(21 \%)$ tienen una presencia significativa en cuanto a su presencia en el currículo. Al igual que en el Marco Curricular en las Bases Curriculares se mantiene el dominio de contenidos de corte tradicional como los contenidos sobre Política tradicional (50\%) y Economía (50\%) que tienen un lugar casi permanente en los temas a tratar cuando se habla de la Historia Reciente. En las 
Bases Curriculares también se observa que el sentido práctico de la enseñanza de la Historia Reciente no logra traducirse completamente en los objetivos de aprendizaje, ya que lo que se propone no logra aún ubicarse en que los estudiantes puedan aprender la Historia Reciente dese una realidad inmediata, cuestión que posible de realizar sobre todo considerando las temáticas de los siguientes objetivos de aprendizaje (MINEDUC 2016):

-Explicar los factores que incidieron en el proceso de recuperación de la democracia durante la década de 1980, considerando la crisis económica, el surgimiento de protestas, la rearticulación del mundo político y el rol mediador de la Iglesia Católica, de organismos de defensa de Derechos Humanos y de la comunidad internacional.

-Analizar la transición a la democracia como un proceso marcado por el plebiscito de 1988, la búsqueda de acuerdos entre el gobierno y la oposición, las reformas constitucionales, las tensiones cívico militares, el consenso generado en torno a la democracia representativa como sistema político y la reivindicación de los Derechos Humanos mediante diversas políticas de reparación.

-Analizar la sociedad chilena posterior a la recuperación de la democracia, considerando la estructura social, la disponibilidad y el acceso a bienes, la infraestructura, los medios y las tecnologías de comunicación, el sistema educacional, el mercado del trabajo y la demanda de derechos de grupos históricamente discriminados, entre otros (MINEDUC 2016: 211-212).

En conclusión, en los currículos de Historia vigentes en las escuelas chilenas en la actualidad podemos establecer que en relación con la Enseñanza de la Historia Reciente esta tiene las características que pueden ubicarla en una perspectiva práctica de la enseñanza de la Historia. Esto se hace notar en las finalidades vinculadas a la formación para la vida en democracia y a la búsqueda del desarrollo personal de los estudiantes. Pese a estas finalidades se mantienen algunos matices que heredados de una perspectiva tradicional de la enseñanza, lo que se manifiesta en el predominio de contenidos políticos y económicos. Y en la construcción de objetivos de aprendizaje que no se ubican en una perspectiva que permita a los estudiantes construir el conocimiento histórico desde su realidad inmediata.

\section{Bibliografía}

Benejam, P. y Pagès, J. 2004. Enseñar y aprender ciencias sociales, geografía e historia en la educación secundaria. Barcelona: Horsori.

Brandes, S. 2002. "Beatniks, hippies, yippies. Orígenes del movimiento estudiantil en Estados Unidos". Movimientos juveniles: De la globalización a la antiglobalización. Feixa, C., Saura, J. y Costa, C. (Eds.). Barcelona: Ariel. 93-109.

Carretero, M. (2007). Documentos de identidad. La construcción de la memoria histórica en un mundo global. Buenos Aires: Paidós.

Carretero, M. y Borrelli, M. 2010. "La historia reciente en la escuela: propuestas para pensar históricamente." La construcción del conocimiento histórico. Enseñanza, narración e identidades. Carretero, M. y Castorina, J. (Eds). Buenos Aires: Paidós. 103 -130.

Cilento, S. 2000. "Retos del habitat urbano venezolano". Espacio Abierto 9 (3): 301-317.

Correa, S. et al. 2001. Historia del siglo XX chileno. Santiago: Editorial Sudamericana.
Crowe, A. R., Hawley, T. S y Brooks, E. W. 2012. "Ways of being a social studies teacher: What are prospective teachers thinking?" Social Studies Research \& Practice 2(7): 50-64.

Cuesta, R. 2000. "Usos y abusos de la educación histórica". Didáctica de las ciencias experimentales y sociales 14: 23-31.

Cuesta, R. 2014. "Memoria, historia y didáctica: los deberes de la educación hacia el pasado, el presente y el futuro." Pedagogía, historia y memoria crítica. Una mirada educativa a los discursos y lugares de la memoria. González F. y Areyuna, B. (Eds.). Santiago: Ediciones on Demand. 27-81.

Fernández, B. 2005. "La radio en los tiempos de la democracia, la globalización y la digitalización". Razón y Palabra 44. Disponible en: http://www.cem.itesm.mx/dacs/publicaciones/logos/actual/ bfernandez.html (consultado en julio de 2005).

Finocchio, S. 2009. "Memoria, historia y educación en la Argentina. De aprender de memoria a enseñar para la memoria de la historia reciente". Història, memòria i ensenyament de la història: perspectives europees i llatinoamericaknes., Pagès, J. y 
González, M. P. (Eds.). Barcelona: Servei de Publicacions. 83-101. Goodman, J. y Adler S. 1985. "Becoming an Elementary Social Studies Teacher: A Study of Perspectives". Theory \& Research in Social Education 13(2): 1-20.

Gómez, S. 2000. "Organizaciones rurales en América Latina (marco para su análisis)". Revista Austral de Ciencias Sociales 4: 27-54.

Grundy, S. 1998. Producto o praxis del currículum. Madrid: Morata.

Hess, D. 2009. Controversy in the classroom. The democratic power of discussion. New York: Routledge.

Hess, D. y McAvoy, P. 2015. The political classroom. Evidence and Ethics in Democratic Education. New York: Routledge.

Kriger, M. 2011. "Enseñanza de la historia reciente como herramienta clave de la educación política." Persona y Sociedad 25(3): 29-52

Lévesque, S. 2009. Thinking historically. Educating students for the twenty-first century. Toronto: University of Toronto Press.

Menanteau, D., Donohue, G. y Fortoul, F. 1977. El rol del sociólogo. Análisis y perspectivas. Valdivia: Universidad Austral de Chile.

Millas, J. 1978. La violencia y sus máscaras. Santiago: Ediciones Aconcagua.

MINEDUC. 2009. Marco Curricular. Chile.

MINEDUC. 2016. Bases Curriculares. $7^{\circ}$ básico a $2^{\circ}$ medio. Disponible en: http://www.curriculumenlineamineduc.cl/605/ articles-36153_recurso_1.pdf (consultado en diciembre 2016)

Pagès, J. 1994. "La didáctica de las ciencias sociales, el currículum y la formación del profesorado." Signos. Teoría y práctica de la educación 13: 38-51.

Pagès, J. 2009. "El desarrollo del pensamiento histórico como requisito para la formación democrática de la ciudadanía."
Reseñas de enseñanza de la historia 7: 69-91.

Pagès, J. y González, M. P. 2009. Història, memòria i ensenyament de la història: perspectives europees llatinoamericanes. Barcelona: Servei de Publicacions.

Santisteban, A. y Pagès, J. 2011. Didáctica del conocimiento del medio social y cultural en la educación Primaria. Ciencias Sociales para aprender, pensar, actuar. Madrid: Editorial Síntesis.

Thornton, S. J. 1991. "Teacher as curricular-instructional gatekeeper in social studies." Handbook of research on social studies teaching and learning. Shaver, J. (Eds.). New York: MacMillan, 237-248.

Tutiaux-Guillon, N. 2011. "Les qüestions socialment vivesm un repte per a la història i la geografia escolars." Les qüestions socialment vives i l'ensenyament de les ciències socials. Pagès $\mathrm{J}$. y Santisteban, A. (Eds.). Barcelona: Servei de Publicacions, 25-39.

Valencia, L. 2013. "La enseñanza del golpe de estado de 1973 y las disputas de la construcción del currículum." Nuevas Dimensiones. Revista Electrónica de Didáctica de las Ciencias Sociales 4: 90-108.

Veneros, D. 2014. "Educación, memoria y trauma cultural: los dilemas de la enseñanza de la historia reciente." Pedagogía, historia y memoria crítica. Una mirada educativa a los discursos y lugares de la memoria. González F. y Areyuna, B. Santiago: Ediciones on Demand, 83-102.

Villalón, G, y Pagès, J. 2013. “¿Quién protagoniza y cómo la historia escolar? La enseñanza de la historia de los otros y las otras en los textos de estudio de Historia de Chile de educación primaria." Clio y asociados 17: 119-36.

2015. "La representación de las y los indígenas en la enseñanza de la historia en la educación básica chilena. El caso de los textos de estudio de historia de Chile." Diálogo Andino 47: 27-36. 
